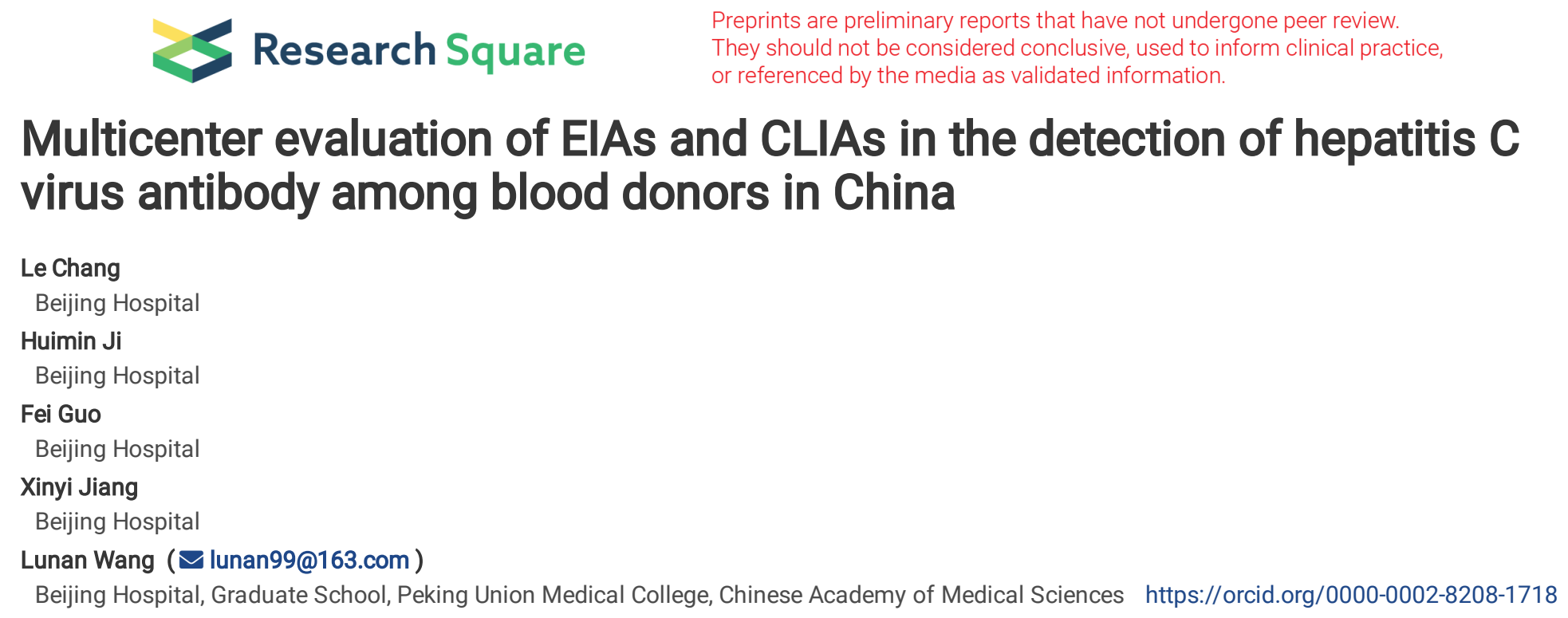

\section{Multicenter evaluation of EIAs and CLIAs in the detection of hepatitis C virus antibody among blood donors in China}




\section{Abstract}

Background. Recent data on the evaluation of screening serological assays on anti-HCV among a large sample of blood donors is lacking. The present study aimed to perform a multicenter evaluation of EIAs and CLIAs for detection of anti-HCV among blood donors in 16 different Chinese blood establishments.

Methods. A total of 1,309 samples containing 582 anti-HCV screening negatives and 727 positives collected from 15 blood establishments all over China. A total of 10 different anti-HCV assays (8 EIAs and 2 CLIAs) were evaluated in 16 different blood centers/banks. Confirmatory testing was performed using recombinant immunoblot assay and HCV RNA tests.

Results. There were 963 true negatives, 261 positives, and 85 indeterminate samples. False positive rate of screening testing was $39.67 \%$ (382/963) and the positive prediction value was only 35.76\% (260/727). Roche and InTec had the highest sensitivity (98.47\%) and KHB and Wantai (indirect EIA) had the highest specificity (99.23\%). Ortho/Abbott together with InTec could detect all the true positives. Among samples with discrepant results by different assays, indeterminate samples showed quite different $\mathrm{S} / \mathrm{CO}$ ratios detected by Roche compared with confirmed positives (4.84 vs 19.36, $\mathrm{P}<0.0001)$, and higher signal to cutoff $(\mathrm{S} / \mathrm{CO})$ ratios than confirmed negatives $(4.84$ vs $2.94, \mathrm{P}=0.020)$. However, there was no difference among the three groups when detected by Abbott. When S/CO ratio went to 8.2 on Roche and 4.2 on Abbott, the PPV could achieve more than $95 \%$.

Conclusion: False reactive problem of anti-HCV screening should be solved urgently. RIBA indeterminate donations may be a special group, should be further studied.

\section{Background}

Hepatitis C virus (HCV) infection is of growing international concern due to a rising burden of its related cirrhosis, hepatocellular carcinoma (HCC), and liver related death [1-3]. In 2015, the global prevalence of viremia HCV is estimated to be $1.0 \%$, that is to say, approximately 71.1 million population worldwide are suffering from HCV and HCV-related diseases [4], and In China, the proportion is about $0.7 \%$ (more than 9 million people are infected).

$\mathrm{HCV}$ is also a blood-borne virus. Laboratory diagnosis of HCV infection is usually made on the basis of the detection of circulating antibodies and/ or HCV RNA [5]. Serological tests are of great importance to preventing transfusion transmitted infections, especially because large majority of HCV infection are anti-HCV reactive and HCV RNA negative [6]. Enzyme immunoassay (EIA) and chemiluminescent immunoassay (CLIA) are two kinds of widely used serological screening methods. In China, serological screening strategy of HCV infection is to adopt two different anti-HCV EIAs that are approved by Chinese Food and Drug Administration (CFDA) and inconsistent results followed by twice detection using the reactive one. When any of the two EIAs is reactive, the donor plasma is disqualified regardless of the NAT results and the donor is deferred for safety [7]. CLIA, which has gradually raised in recent years, can automatically and quickly detect serum/plasma anti-HCV with higher sensitivity and specificity but haven't been used in Chinese blood centers/banks.

However, recent data on evaluation of screening serological assays on anti-HCV among a large sample of blood donors are lacking. Such data are important to help us to analysis and find difference among different assays and improve assays' performance. The present study aimed to perform a multicenter evaluation of enzyme immunoassays and chemiluminescent immunoassays for detection of antibodies against hepatitis $\mathrm{C}$ virus among blood donors in 16 different Chinese blood centers/banks.

\section{Methods \\ Plasma panel}

A total of 1,309 plasma samples collected by 15 different blood centers or blood banks during Apr. 2015 to Sept. 2015 , including Changchun (abbreviated as CC), Chongqing (CQ), Hebei (HB), Heilongjiang (HLJ), Henan (HN), Heze (HZ), Jining (JN), Jiangsu (JS), Kuming (KM), Liaoning (LN), Shandong (SD), Shenzhen (SZ), Tianjin (TJ), Tongzhou (TZ), Xiangyang (XY) blood center/blood bank, among which there were 582 anti-HCV negative( HBsAg, anti-HCV, anti-HIV and anti-TP all negative by both two screening EIAs in the enrolled laboratories, but discarded due to ALT $\geq$ $50 \mathrm{U} / \mathrm{L}$, which is the blood screening strategy in China) and 727 anti-HCV positive samples by one or two EIAs (If only one EIA was positive then the donation would be tested twice with the assay again. If any was positive the donation would be defined as positive by screening test) in blood stations. All these samples were sent to National Center for Clinical Laboratories (NCCL), split, numbered, stored at $-40{ }^{\circ} \mathrm{C}$ and then sent back to all the participants. The transportation of the plasma panel was below $4{ }^{\circ} \mathrm{C}$ and within 3 days.

\section{Study design}

16 different blood centers or blood banks over China were participated into this multicenter evaluation, including CC, CQ, Cangzhou (CZ), HB, HLJ, HN, JN, JS, KM, LN, SD, Shanghai (SH), SZ, TJ, TZ, XY blood center/bank. The moment all these stations above received the panel, they used one or 
two different EIAs automatically to test within 3 days. Each sample was only tested once by one assay. All the results were sent back to NCCL within 10 days. Besides, NCCL tested all the samples once by two different CLIA assays simultaneously. According to the confirmatory process (Fig. 1), NCCL finished to confirm all the panel samples within one month. All results were performed according to the manufacturers' instructions.

\section{Evaluated assays}

there were 10 different commercially available anti-HCV assays in this study included KHB Diagnostic Kit for Antibody to Hepatitis C virus (Shanghai Kehua Bioengineering co., Shanghai, China), InTec Diagnostic Kit for Antibody to Hepatitis C virus (InTec Products, Inc., Xiamen, China), ORTHO HCV Version 3.0 ELISA (Ortho-Clinical Diagnostics, Raritan, New Jersey), Livzon Diagnostic Kit for Antibody to Hepatitis C virus (indirect) (Zhuhai Livzon Diagnostics Inc., Zhuhai, China), Wantai Diagnostic Kit for Antibody to Hepatitis C virus (indirect) (Beijing Wantai Biological Pharmacy, Beijing, China), Wantai Diagnostic Kit for Antibody to Hepatitis C virus (sandwich) (Beijing Wantai Biological Pharmacy, Beijing, China), Livzon Diagnostic Kit for Antibody to Hepatitis C virus (sandwich) Zhuhai Livzon Diagnostics Inc., Zhuhai, China), Murex anti-HCV (version 4.0) (Diasorin, Saluggia, Italy), ARCHITECT anti-HCV (Abbott, Illinois, US), Elecsys Anti-HCV II (Roche Diagnostics GmbH, Penzberg, Germany). Technical specifications of evaluated assays see Table 1.

Table 1

Technical specifications of evaluated assays and confirmatory assay

\begin{tabular}{|c|c|c|c|}
\hline Abbreviations of assays & Test format + & Antigens & Stations \\
\hline $\mathrm{KHB}$ & EIA, indirect & core, non-structure antigen & $\mathrm{CQ}, \mathrm{HLJ}, \mathrm{HN}, \mathrm{XY}$ \\
\hline InTec & EIA, indirect & $\mathrm{N} / \mathrm{M} \ddagger$ & $\mathrm{CC}, \mathrm{HB}, \mathrm{JN}, \mathrm{JS}, \mathrm{XY}$ \\
\hline Ortho & EIA, indirect & core(c22-3), NS3 + NS4(c200), NS5 & $\mathrm{CQ}, \mathrm{SZ}, \mathrm{TJ}$ \\
\hline Livzon (indirect) & EIA, indirect & $\mathrm{N} / \mathrm{M}$ & CC, JS, SD \\
\hline Wantai (indirect) & EIA, indirect & $\mathrm{N} / \mathrm{M}$ & CZ, LN, SD \\
\hline Wantai (sandwich) & EIA, sandwich & $\mathrm{N} / \mathrm{M}$ & $\mathrm{CZ}, \mathrm{HB}, \mathrm{HLJ}, \mathrm{HN}, \mathrm{LN}, \mathrm{TJ}$ \\
\hline Livzon (sandwich) & EIA, sandwich & core, NS3, etc. & JN, LN \\
\hline Murex & EIA, indirect & core, NS3, NS4, NS5 & KM, SH, TZ \\
\hline Roche & ECLIA, sandwich & core, NS3, NS4 & NCCL \\
\hline Abbott & CMIA, indirect & core, NS3(33c), NS4(c100-3) & NCCL \\
\hline Mikrogen & RIBA & core1, core2, helicase, NS3, NS4, NS5 & NCCL \\
\hline \multicolumn{4}{|l|}{ Note: } \\
\hline \multicolumn{4}{|c|}{$\begin{array}{l}\text { † EIA: enzyme immunoassay; ECLIA: electrochemiluminescence immunoassay; CMIA: chemiluminescent microparticle immunoassay; RIBA } \\
\text { recombinant immunobinding assay. }\end{array}$} \\
\hline
\end{tabular}

\section{Confirmatory tests}

According to the confirmatory algorithm (Fig. 1), part of discrepant results of samples among 10 assays were then subjected to confirmatory testing with anti-HCV recombinant immunoblot assay (RIBA, Mikrogen Diagnostic, Martinsried, Germany), which expressed the results as positive, negative, and indeterminate. All results were performed according to the manufacturers' instructions. Technical specifications of confirmatory assay see Table 1.

\section{Data and Statistical analysis}

Assay's results of each sample: one sample tested by the same assay showed consistent results in different stations, the assay's result of this sample was the same as the consistent result. When different stations showed different results, take the majority results as the assay's result of this sample. When the number of positives equaled negatives, then take the average signal to cutoff value (S/CO) as the assay's result.

Confirmatory results of each sample: Samples showing consistent results among all of the assays would be considered as either true positive or true negative. Sample tested positive by Roche and Abbott but with discrepant results by EIAs and simultaneously with S/CO $\geq 5.0$ by Abbott would also be considered as true positive [5]. Other samples with discrepant results were further confirmed by RIBA. Samples with an indeterminate outcome for immunoblotting were retested HCV RNA individually by cobas TaqScreen MPX Test, version2.0 (Roche Molecular Diagnostics, Rotkreuz, Switzerland). All the indeterminate samples were excluded from the interassay comparisons. 
The sensitivity, specificity, Youden's index (The formula was below) and Kappa coefficient of each assay and combined assays, and receiver operating characteristic curve (ROC) and area under curve (AUC) were calculated by SPSS software (v.21.0). Results were analyzed with One-Way ANOVA analysis and Chi-square test. $P<0.05$ was considered as statistically significance.

Sensitivity $=$ true-positives $/$ (true-positives + false-negatives) $\times 100 \%$;

Specificity $=$ true-negatives $/$ (true-negatives + false-positives) $\times 100 \%$;

Youden's index $=$ Sensitivity + Specificity- 1

\section{Results}

\section{Confirmatory results of plasma panel}

In 1,309 plasma samples, 963 were true negative, 261 were true positive and 85 were indeterminate. Among all the true negatives, 724 ( $724 / 963$, 75.18\%) were detected negative by all 10 assays. 209 positives were detected positive by all assays. 376 samples showed discrepant results, among which 36 were reactive by two CLIAs and S/CO ratio of 15 samples by both two assays were above 5.0, which were also considered as true positives. 361 samples were subjected to confirmatory testing, only 37 were true positive and 85 were indeterminate (HCV RNA were also undetected in the indeterminate samples). The final results and validation algorithm see Fig. 1.

In 727 anti-HCV initial reactive samples only 260 were true positive, indicating the average positive prediction value (PPV) of anti-HCV screening tests was only $35.76 \%$ (260/727) and the average false positive rate was as high as $52.54 \%(382 / 727)$. Besides, we also found one true positive samples from Liaoning blood center were undetected by screening assays. Sample source and confirmatory results see Table 2 .

Table 2

Sample source and confirmatory results

\begin{tabular}{|c|c|c|c|c|c|c|c|c|c|}
\hline \multirow{2}{*}{$\begin{array}{l}\text { Samples } \\
\text { source } \\
\text { (stations) }\end{array}$} & \multicolumn{3}{|c|}{$\begin{array}{l}\text { anti-HCV- } \\
\text { by screening assays }\end{array}$} & \multicolumn{4}{|c|}{$\begin{array}{l}\text { anti-HCV+ } \\
\text { by screening assays }\end{array}$} & \multirow[t]{2}{*}{ False negative \% } & \multirow[t]{2}{*}{ False positive $\%$} \\
\hline & total & $-t$ & $+t$ & total & $-t$ & $+t$ & $+/-t$ & & \\
\hline NCCL & 0 & 0 & 0 & 129 & 87 & 33 & 9 & 0.00 & 67.44 \\
\hline $\mathrm{CC}$ & 11 & 11 & 0 & 35 & 9 & 23 & 3 & 0.00 & 25.71 \\
\hline $\mathrm{CQ}$ & 22 & 22 & 0 & 48 & 24 & 16 & 8 & 0.00 & 50.00 \\
\hline $\mathrm{HB}$ & 18 & 18 & 0 & 48 & 31 & 12 & 5 & 0.00 & 64.58 \\
\hline HLJ & 76 & 76 & 0 & 30 & 12 & 16 & 2 & 0.00 & 40.00 \\
\hline $\mathrm{HN}$ & 111 & 111 & 0 & 66 & 31 & 23 & 12 & 0.00 & 46.97 \\
\hline $\mathrm{HZ}$ & 21 & 21 & 0 & 5 & 2 & 3 & 0 & 0.00 & 40.00 \\
\hline JN & 0 & 0 & 0 & 4 & 4 & 0 & 0 & 0.00 & 100.00 \\
\hline JS & 50 & 50 & 0 & 50 & 33 & 14 & 3 & 0.00 & 66.00 \\
\hline KM & 15 & 15 & 0 & 11 & 3 & 6 & 2 & 0.00 & 27.27 \\
\hline LN & 50 & 49 & 1 & 37 & 12 & 19 & 6 & 2.00 & 32.43 \\
\hline SD & 50 & 50 & 0 & 49 & 23 & 21 & 5 & 0.00 & 46.94 \\
\hline SZ & 50 & 50 & 0 & 50 & 30 & 13 & 7 & 0.00 & 60.00 \\
\hline TJ & 49 & 49 & 0 & 101 & 43 & 42 & 16 & 0.00 & 42.57 \\
\hline $\mathrm{TZ}$ & 50 & 50 & 0 & 35 & 20 & 11 & 4 & 0.00 & 57.14 \\
\hline$X Y$ & 9 & 9 & 0 & 29 & 18 & 8 & 3 & 0.00 & 62.07 \\
\hline Total & 582 & 581 & 1 & 727 & 382 & 260 & 85 & 0.17 & 52.54 \\
\hline
\end{tabular}

\section{Performance of evaluated assays}


On the basis of confirmatory results and assay's results of 963 true negatives and 261 true positives, we calculated the sensitivity, specificity, Youden's index and Kappa Coefficient. The results showed assays' sensitivity varied from 91.19-98.47\%, InTec and Roche owned the highest sensitivity (98.47\%), while sensitivity of Abbott was the lowest (91.19\%). Since in China as a routine practice, two different kinds of ElAs are used simultaneously, we calculated the combined performance index between two different assays. The combined sensitivity showed that when we use InTec and Ortho, 100\% positives could be detected. Similarly, different assays showed different specificity (92.94\%-98.23\%), KHB was the highest, while InTec was the lowest. When Wantai (sandwich) and Wantai (indirect) were used together, we could get the highest combined specificity (97.92\%). Youden's index, a way of summarizing the performance of a diagnostic test, equals sensitivity plus specificity minus 1 . Roche got the highest index (96.08\%) and the two EIAs, KHB and Livzon (indirect), owned the highest combined Youden's index. Results of Kappa coefficient, a statistic which measures inter-rater agreement, indicated that Wantai (indirect) had perfect agreement with confirmatory results, and Wantai (indirect) and Livzon (sandwich) together owned the highest kappa coefficient. As a whole, CLIAs didn't perform better than EIAs on the detection of anti-HCV in this study. All the detail results see Table 3. 
Table 3

Performance index of different assays

\begin{tabular}{|c|c|c|c|c|c|c|c|c|c|c|}
\hline $\begin{array}{l}\text { Sensitivity } \\
(\%)\end{array}$ & KHB & InTec & Ortho & $\begin{array}{l}\text { Livzon } \\
\text { (indirect) }\end{array}$ & $\begin{array}{l}\text { Wantai } \\
\text { (indirect) }\end{array}$ & $\begin{array}{l}\text { Wantai } \\
\text { (sandwich) }\end{array}$ & $\begin{array}{l}\text { Livzon } \\
\text { (sandwich) }\end{array}$ & Murex & Roche & Abbott \\
\hline $\mathrm{KHB}$ & $95.02 \%$ & & & & & & & & & \\
\hline InTec & $99.23 \%$ & $98.47 \%$ & & & & & & & & \\
\hline Ortho & $98.08 \%$ & $100.00 \%$ & $92.72 \%$ & & & & & & & \\
\hline $\begin{array}{l}\text { Livzon } \\
\text { (indirect) }\end{array}$ & $99.23 \%$ & $98.85 \%$ & $99.23 \%$ & $91.95 \%$ & & & & & & \\
\hline $\begin{array}{l}\text { Wantai } \\
\text { (indirect) }\end{array}$ & $98.85 \%$ & $98.85 \%$ & $99.62 \%$ & $99.23 \%$ & $97.70 \%$ & & & & & \\
\hline $\begin{array}{l}\text { Wantai } \\
\text { (sandwich) }\end{array}$ & $98.47 \%$ & $98.85 \%$ & $99.23 \%$ & $99.23 \%$ & $97.70 \%$ & $97.32 \%$ & & & & \\
\hline $\begin{array}{l}\text { Livzon } \\
\text { (sandwich) }\end{array}$ & $98.47 \%$ & $98.85 \%$ & $99.23 \%$ & $99.23 \%$ & $97.70 \%$ & $97.32 \%$ & $97.32 \%$ & & & \\
\hline Murex & $96.17 \%$ & $99.23 \%$ & $96.55 \%$ & $99.23 \%$ & $98.08 \%$ & $97.70 \%$ & $97.70 \%$ & $92.72 \%$ & & \\
\hline Roche & $98.85 \%$ & $98.85 \%$ & $100.00 \%$ & $99.23 \%$ & $98.47 \%$ & $98.50 \%$ & $98.47 \%$ & $98.85 \%$ & $98.47 \%$ & \\
\hline Abbott & $96.93 \%$ & $100.00 \%$ & $94.25 \%$ & $98.85 \%$ & $99.62 \%$ & $99.23 \%$ & $99.23 \%$ & $95.79 \%$ & $99.62 \%$ & $91.19 \%$ \\
\hline $\begin{array}{l}\text { Specificity } \\
(\%)\end{array}$ & $\mathrm{KHB}$ & InTec & Ortho & $\begin{array}{l}\text { Livzon } \\
\text { (indirect) }\end{array}$ & $\begin{array}{l}\text { Wantai } \\
\text { (indirect) }\end{array}$ & $\begin{array}{l}\text { Wantai } \\
\text { (sandwich) }\end{array}$ & $\begin{array}{l}\text { Livzon } \\
\text { (sandwich) }\end{array}$ & Murex & Roche & Abbott \\
\hline $\mathrm{KHB}$ & $98.23 \%$ & & & & & & & & & \\
\hline InTec & $91.80 \%$ & $92.94 \%$ & & & & & & & & \\
\hline Ortho & $89.72 \%$ & $84.53 \%$ & $90.13 \%$ & & & & & & & \\
\hline $\begin{array}{l}\text { Livzon } \\
\text { (indirect) }\end{array}$ & $92.21 \%$ & $87.23 \%$ & $84.74 \%$ & $93.15 \%$ & & & & & & \\
\hline $\begin{array}{l}\text { Wantai } \\
\text { (indirect) }\end{array}$ & $96.88 \%$ & $91.80 \%$ & $88.79 \%$ & $92.32 \%$ & $98.23 \%$ & & & & & \\
\hline $\begin{array}{l}\text { Wantai } \\
\text { (sandwich) }\end{array}$ & $96.68 \%$ & $91.59 \%$ & $88.58 \%$ & $92.00 \%$ & $97.80 \%$ & $98.03 \%$ & & & & \\
\hline $\begin{array}{l}\text { Livzon } \\
\text { (sandwich) }\end{array}$ & $96.78 \%$ & $91.69 \%$ & $88.79 \%$ & $92.32 \%$ & $97.92 \%$ & $97.72 \%$ & $98.13 \%$ & & & \\
\hline Murex & $95.85 \%$ & $90.55 \%$ & $88.06 \%$ & $91.07 \%$ & $95.74 \%$ & $95.53 \%$ & $95.64 \%$ & $97.20 \%$ & & \\
\hline Roche & $96.26 \%$ & $91.17 \%$ & $88.27 \%$ & $91.80 \%$ & $97.40 \%$ & $97.30 \%$ & $97.40 \%$ & $95.22 \%$ & $97.61 \%$ & \\
\hline Abbott & $92.94 \%$ & $86.71 \%$ & $87.75 \%$ & $87.54 \%$ & $91.90 \%$ & $91.69 \%$ & $91.80 \%$ & $91.38 \%$ & $91.48 \%$ & $93.25 \%$ \\
\hline $\begin{array}{l}\text { Youden's } \\
\text { index }\end{array}$ & $\mathrm{KHB}$ & InTec & Ortho & $\begin{array}{l}\text { Livzon } \\
\text { (indirect) }\end{array}$ & $\begin{array}{l}\text { Wantai } \\
\text { (indirect) }\end{array}$ & $\begin{array}{l}\text { Wantai } \\
\text { (sandwich) }\end{array}$ & $\begin{array}{l}\text { Livzon } \\
\text { (sandwich) }\end{array}$ & Murex & Roche & Abbott \\
\hline $\mathrm{KHB}$ & 0.933 & & & & & & & & & \\
\hline InTec & 0.910 & 0.914 & & & & & & & & \\
\hline Ortho & 0.878 & 0.845 & 0.829 & & & & & & & \\
\hline $\begin{array}{l}\text { Livzon } \\
\text { (indirect) }\end{array}$ & 0.914 & 0.861 & 0.840 & 0.851 & & & & & & \\
\hline $\begin{array}{l}\text { Wantai } \\
\text { (indirect) }\end{array}$ & 0.957 & 0.906 & 0.884 & 0.915 & 0.959 & & & & & \\
\hline $\begin{array}{l}\text { Wantai } \\
\text { (sandwich) }\end{array}$ & 0.951 & 0.904 & 0.878 & 0.912 & 0.955 & 0.953 & & & & \\
\hline $\begin{array}{l}\text { Livzon } \\
\text { (sandwich) }\end{array}$ & 0.952 & 0.905 & 0.880 & 0.915 & 0.956 & 0.950 & 0.954 & & & \\
\hline Murex & 0.920 & 0.898 & 0.846 & 0.903 & 0.938 & 0.932 & 0.933 & 0.899 & & \\
\hline Roche & 0.951 & 0.900 & 0.883 & 0.910 & 0.959 & 0.958 & 0.959 & 0.941 & 0.961 & \\
\hline
\end{tabular}




\begin{tabular}{|c|c|c|c|c|c|c|c|c|c|c|}
\hline $\begin{array}{l}\text { Sensitivity } \\
\text { (\%) }\end{array}$ & KHB & InTec & Ortho & $\begin{array}{l}\text { Livzon } \\
\text { (indirect) }\end{array}$ & $\begin{array}{l}\text { Wantai } \\
\text { (indirect) }\end{array}$ & $\begin{array}{l}\text { Wantai } \\
\text { (sandwich) }\end{array}$ & $\begin{array}{l}\text { Livzon } \\
\text { (sandwich) }\end{array}$ & Murex & Roche & Abbott \\
\hline Abbott & 0.899 & 0.867 & 0.820 & 0.864 & 0.915 & 0.909 & 0.910 & 0.872 & 0.911 & 0.844 \\
\hline $\begin{array}{l}\text { Kappa } \\
\text { Coefficient }\end{array}$ & KHB & InTec & Ortho & $\begin{array}{l}\text { Livzon } \\
\text { (indirect) }\end{array}$ & $\begin{array}{l}\text { Wantai } \\
\text { (indirect) }\end{array}$ & $\begin{array}{l}\text { Wantai } \\
\text { (sandwich) }\end{array}$ & $\begin{array}{l}\text { Livzon } \\
\text { (sandwich) }\end{array}$ & Murex & Roche & Abbott \\
\hline KHB & 0.927 & & & & & & & & & \\
\hline InTec & 0.822 & 0.839 & & & & & & & & \\
\hline Ortho & 0.776 & 0.700 & 0.749 & & & & & & & \\
\hline $\begin{array}{l}\text { Livzon } \\
\text { (indirect) }\end{array}$ & 0.830 & 0.737 & 0.698 & 0.801 & & & & & & \\
\hline $\begin{array}{l}\text { Wantai } \\
\text { (indirect) }\end{array}$ & 0.923 & 0.819 & 0.769 & 0.832 & 0.945 & & & & & \\
\hline $\begin{array}{l}\text { Wantai } \\
\text { (sandwich) }\end{array}$ & 0.916 & 0.816 & 0.763 & 0.826 & 0.936 & 0.938 & & & & \\
\hline $\begin{array}{l}\text { Livzon } \\
\text { (sandwich) }\end{array}$ & 0.918 & 0.818 & 0.767 & 0.832 & 0.938 & 0.931 & 0.940 & & & \\
\hline Murex & 0.883 & 0.799 & 0.737 & 0.808 & 0.893 & 0.887 & 0.889 & 0.889 & & \\
\hline Roche & 0.909 & 0.808 & 0.762 & 0.822 & 0.931 & 0.929 & 0.931 & 0.887 & 0.936 & \\
\hline Abbott & 0.829 & 0.736 & 0.717 & 0.743 & 0.826 & 0.820 & 0.822 & 0.792 & 0.818 & 0.798 \\
\hline
\end{tabular}

\section{Indeterminate samples}

There were 85 samples indeterminate by RIBA and HCV RNA undetected. We further explored the antigen distribution and found there were 6 different formats: core1 single, helicase single, helicase/NS3, NS3/NS4, NS4/NS5 and NS4/NS5, among which core1 single was the majority $(53 / 85,62.35 \%)$, next was helicase single (17/85, 20\%). Only 3 samples belong to last three formats (Table 4). Different assays showed quite different results on these indeterminate samples: of 1,309 samples, Abbott had 45 positives confirmed as indeterminate, while Wantai (sandwich) and Murex got only 25 indeterminate. In the aspect of antigen distribution among different assays, most assays showed similar results as the total, except Murex. Helicase single in Murex positive indeterminate samples accounted for a main proportion (40\%), next is core1 single (32\%) and Helicase/NS3 format (28\%).

Table 4

Antigen distribution on indeterminate samples

\begin{tabular}{|c|c|c|c|c|c|c|c|c|c|c|c|}
\hline Antigens & KHB & InTec & Ortho & $\begin{array}{l}\text { Livzon } \\
\text { (indirect) }\end{array}$ & $\begin{array}{l}\text { Wantai } \\
\text { (indirect) }\end{array}$ & $\begin{array}{l}\text { Wantai } \\
\text { (sandwich) }\end{array}$ & $\begin{array}{l}\text { Livzon } \\
\text { (sandwich) }\end{array}$ & Murex & Roche & Abbott & Total \\
\hline \multirow[t]{2}{*}{ core1 } & 15 & 26 & 24 & 24 & 24 & 23 & 25 & 8 & 26 & 24 & 53 \\
\hline & $51.72 \%$ & $70.27 \%$ & $58.54 \%$ & $63.16 \%$ & $92.31 \%$ & $92.00 \%$ & $92.59 \%$ & $32.00 \%$ & $89.66 \%$ & $53.33 \%$ & $62.35 \%$ \\
\hline \multirow[t]{2}{*}{ Helicase } & 9 & 5 & 7 & 7 & 2 & 1 & 1 & 10 & 1 & 13 & 17 \\
\hline & $31.03 \%$ & $13.51 \%$ & $17.07 \%$ & $18.42 \%$ & $7.69 \%$ & $4.00 \%$ & $3.70 \%$ & $40.00 \%$ & $3.45 \%$ & $28.89 \%$ & $20.00 \%$ \\
\hline \multirow[t]{2}{*}{ Helicase/NS3 } & 5 & 3 & 7 & 6 & 0 & 1 & 1 & 7 & 2 & 8 & 12 \\
\hline & $17.24 \%$ & $8.11 \%$ & $17.07 \%$ & $15.79 \%$ & $0.00 \%$ & $4.00 \%$ & $3.70 \%$ & $28.00 \%$ & $6.90 \%$ & $17.78 \%$ & $14.12 \%$ \\
\hline \multirow[t]{2}{*}{ NS3/NS4 } & 0 & 1 & 1 & 0 & 0 & 0 & 0 & 0 & 0 & 0 & 1 \\
\hline & $0.00 \%$ & $2.70 \%$ & $2.44 \%$ & $0.00 \%$ & $0.00 \%$ & $0.00 \%$ & $0.00 \%$ & $0.00 \%$ & $0.00 \%$ & $0.00 \%$ & $1.18 \%$ \\
\hline \multirow[t]{2}{*}{ NS3/NS5 } & 0 & 1 & 1 & 1 & 0 & 0 & 0 & 0 & 0 & 0 & 1 \\
\hline & $0.00 \%$ & $2.70 \%$ & $2.44 \%$ & $2.63 \%$ & $0.00 \%$ & $0.00 \%$ & $0.00 \%$ & $0.00 \%$ & $0.00 \%$ & $0.00 \%$ & $1.18 \%$ \\
\hline \multirow[t]{2}{*}{ NS4/NS5 } & 0 & 1 & 1 & 0 & 0 & 0 & 0 & 0 & 0 & 0 & 1 \\
\hline & $0.00 \%$ & $2.70 \%$ & $2.44 \%$ & $0.00 \%$ & $0.00 \%$ & $0.00 \%$ & $0.00 \%$ & $0.00 \%$ & $0.00 \%$ & $0.00 \%$ & $1.18 \%$ \\
\hline Total & 29 & 37 & 41 & 38 & 26 & 25 & 27 & 25 & 29 & 45 & 85 \\
\hline
\end{tabular}

The comparison of RIBA band intensities between RIBA indeterminate and RIBA positive samples was shown in Table 5, which indicated that the two groups had quite different antigen proportion $(P<0.0001)$ : there was no core2 reactives in RIBA indeterminate group and much more NS3 
reactives, while in RIBA positive group core2 reactives accounted for 51.35\% (19/37) and more NS4 reactives. Among core1 reactive samples, the RIBA positive had a relatively higher band intensity $(P<0.0001)$.

Table 5

Comparison of RIBA band intensities for RIBA-indeterminate and -positive samples

\begin{tabular}{|c|c|c|c|c|c|c|}
\hline & \multicolumn{6}{|c|}{ RIBA indeterminate } \\
\hline & core1 & core2 & helicase & NS3 & NS4 & NS5 \\
\hline $1+$ & 17 & 0 & 5 & 13 & 2 & 2 \\
\hline $2+$ & 33 & 0 & 20 & 1 & 0 & 0 \\
\hline $3+$ & 3 & 0 & 4 & 0 & 0 & 0 \\
\hline total & 53 & 0 & 29 & 14 & 2 & 2 \\
\hline \multirow[t]{3}{*}{$\%$} & 62.35 & 0.00 & 34.12 & 16.47 & 2.35 & 2.35 \\
\hline & \multicolumn{6}{|c|}{ RIBA positive † } \\
\hline & core $1 \neq$ & core2 & helicase & NS3 & NS4 & NS5 \\
\hline $1+$ & 3 & 19 & 4 & 1 & 15 & 1 \\
\hline $2+$ & 26 & 0 & 1 & 0 & 1 & 0 \\
\hline $3+$ & 8 & 0 & 0 & 0 & 0 & 0 \\
\hline total & 37 & 19 & 5 & 1 & 16 & 1 \\
\hline$\%$ & 100.00 & 51.35 & 13.51 & 2.70 & 43.24 & 2.70 \\
\hline \multicolumn{7}{|l|}{ Note: } \\
\hline \multicolumn{7}{|c|}{ † Comparison of proportion of different bands. The difference is statistically significant, $P<0.0001$. } \\
\hline
\end{tabular}

Besides, we also analyzed the S/CO ratios of Roche and Abbott among RIBA negative, indeterminate and positive samples. There was statistically significant between RIBA indeterminate and positive (4.84 vs 19.36, $P<0.0001)$, between negative and positive $(2.94$ vs $19.36, P<0.0001)$ and between RIBA determinate and negative (4.84 vs 2.94, $P=0.020)$ detected by Roche. However, we didn't find any difference among the three groups when detected by Abbott (positive 1.40 vs indeterminate 1.74 vs negative $1.55, P=0.567$ ) (Fig. 2 )

\section{PPV of CLIAs on different S/CO ratios}

In order to determine the relationship between PPV and S/CO ratio on two CLIAs, we analyzed the assays results when different S/CO ratios were as the cutoff values of the two CLIAs. We found that when S/CO ratio went to 1.0, Roche's PPV on detection of anti-HCV was $92.09 \%$ (256/278), and Abbott was only 78.55\% (238/303). When PPV was above 95\% (246/258 on Roche, 196/205 on Abbott), cutoff S/CO ratios need to be more than 8.2 on Roche, and more than 4.2 on Abbott (Table 6). When PPV was 100\% (65/65 on Roche, $177 / 177$ on Abbott), cutoff S/CO ratios need to be 46.8 on Roche, and 8.0 on Abbott. Results of ROC and AUC indicated that the two assays both showed a good AUC: Roche was 0.994 and Abbott was 0.984 . However, the best S/CO was quite different on the two: S/CO ratio $=1.515$ was the best cutoff for Roche, on this S/CO the assay's sensitivity was $98.08 \%$ and the specificity was $97.82 \%$. Since the relatively low sensitivity of Abbott, the best S/CO was only 0.385 (sensitivity $=99.23 \%$, specificity $=86.50 \%$ ). 
Table 6

Relationship between S/CO and PPV

\begin{tabular}{|llll|}
\hline Roche & \multicolumn{3}{|c|}{ Abbott } \\
\hline S/CO & PPV & S/CO & PPV \\
\hline 1.0 & $92.09 \%$ & 1.0 & $78.55 \%$ \\
\hline 3.0 & $92.70 \%$ & 3.0 & $91.07 \%$ \\
\hline 5.0 & $94.03 \%$ & 4.0 & $94.26 \%$ \\
\hline 7.0 & $94.64 \%$ & 4.2 & $95.61 \%$ \\
\hline 8.2 & $95.35 \%$ & 5.0 & $97.93 \%$ \\
\hline 15.0 & $96.65 \%$ & 6.0 & $98.91 \%$ \\
\hline 25.0 & $97.24 \%$ & 6.2 & $99.45 \%$ \\
\hline 35.0 & $99.06 \%$ & 7.0 & $99.45 \%$ \\
\hline 46.8 & $100.00 \%$ & 8.0 & $100.00 \%$ \\
\hline
\end{tabular}

\section{Discussion}

False positive reactions in anti-HCV screening assays is a well-known problem and an obstacle to improve assays' performance all along. In the present study, we found that the average false positive rate of anti-HCV screening assays was as high as $52.54 \%$, which meant large amount of donations were discarded and qualified donors were deferred unfortunately. It is reported in Uganda, 7.6\% (76/1000) were serologically reactive by Ortho, but none were confirmed [8]. Similarly, Schroter M, et al [9]also found false positive results by widely used anti-HCV EIAs were at least 10\%, which, compared to other virus screening assays, was unacceptable.

Whereas the false positive rate in this study was much higher than the two reports above, which might partly result from the serological screening strategy in China. Use of two EIAs no doubt would relatively increase the sensitivity but sacrifice the specificity to a certain extent. In the next place, samples used in screening tests were directly from blood collection tubes, while samples in the plasma panel were from plasma bag, which contained about $20 \%$ blood preserving fluid, so that diluting the samples and resulting in missing detection of some weak positives. Besides, researchers also found in low risk populations, $40-50 \%$ of screening reactives were negative in supplemental immunoblot tests, which was similar to our results [10].

Secondly, performance analysis of 10 different assays indicated that generally all assays' performance was worse than reported [11-19] or claimed by their instructions, which may be mainly due to sample source. Large amount of false positive samples increased the difficulty of testing a lot and false positive results of an assay during the first screening are likely to be false positives again. Specifically, Roche got the highest sensitivity (98.47\%) and Youden's index (0.961) but specificity (97.61\%) was lower than four EIAs (KHB 98.23\%, Wantai (indirect) 98.23\%, Wantai (sandwich) 98.03\% and Livzon (sandwich) 98.13\%). The other CLIA, Abbott didn't perform well: it had the lowest sensitivity, but its results were highly comparable to those of Ortho, which also has been shown in other studies [17-20]. Therefore, Two CLIAs especially in the respect of specificity didn't show much better performance than EIAs, no matter imported assays or domestic ones.

Since the strategy of two ElAs together using in China, we then analyzed the combined performance of any two assays. Interestingly, not two assays both with good sensitivity had the best combined sensitivity, but Ortho/Abbott together with InTec had a $100 \%$ sensitivity indicating these two assays just made up for each other's missing detection. In the terms of specificity, though use of two assays would definitely decrease detection specificity, we found that Wantai (indirect) together with Livzon (sandwich) got a $97.80 \%$ specificity, even better than only using one assay (InTec 92.94\%, Ortho 90.13\%, Livzon(indirect) 93.15\%, Murex 97.20\%, Roche 97.61\%, Abbott 93.25\%).

Analysis of assays' performance also suggested that confirmation of positive HCV screening results by supplemental tests is necessary. Usually, we use RIBA and/or NAT as a way to confirm screening reactive samples. However, RIBA as a confirmatory method for serological screening test of $\mathrm{HCV}$, is too expensive and laborious to be used in every laboratory in China and these confirmatory tests often gives many RIBA indeterminate and/or HCV RNA negative results, indicating the donor may be a resolved HCV infection, infection with a different genotype, early seroconversion, occult HCV infection or just non-specific reactivity [21], which makes us much difficult to estimate the donations' true status. Like our results, we got $85(6.49 \%, 85 / 1,309)$ RIBA indeterminate/HCV RNA negative samples, and most $(53 / 85,62.35 \%)$ were due to isolated reactivity to antigens from the core1 region of the HCV genome. We also found these indeterminate samples showed quite different average S/CO ratio detected by Roche compared with RIBA positives (4.84 vs $19.36, P<0.0001)$, higher S/CO ratios than RIBA negatives $(4.84$ vs $2.94, P=0.020)$. Similarly to Kiely P's results [21], the antigen distribution and band intensity were different between indeterminate samples and RIBA positives. All these results indicated RIBA indeterminate samples were a special group different from negative and positives. 
Finally, we talked about the relationship between PPV and S/CO ratio on two CLIAs. When S/CO went to 8.2 on Roche and 4.2 on Abbott, the PPV could achieve more than $95 \%$. The S/CO ratio of ARCHITECT anti-HCV established for FDA-approved is more than 5.0 [5], similar to our results. But there is no such approved ratio on Elecsys Anti-HCV II. Some studies suggested that the value predictive of a true positive $\geq 95 \%$ of the time with the assay could be set at an S/CO ratio of 20.0 , much higher than our study $[13,15]$, which meant it was required to verify the value in further study.

\section{Conclusion}

Based on the multicenter evaluation of 10 common used anti-HCV screening assays, false reactive problem of anti-HCV screening should be solved urgently. Assays showed different performance on sensitivity and specificity and CLIAs didn't performed better than EIAs, especially on specificity. RIBA indeterminate donations may be a special group, should be further studied.

\section{Abbreviations}

HCV, Hepatitis C virus; EIA, Enzyme immunoassay; CLIA, Chemiluminescent immunoassay; NCCL National Center for Clinical Laboratories; RIBA:recombinant immunoblot assay.

\section{Declarations}

Ethics approval and consent to participate: The ethics committee of Beijing Hospital has approved the study. The methods in the study were in accordance with the guidelines of the Declaration of Helsinki. Written informed consent at the time of blood donation was obtained from all blood donors participating in this research.

Consent for publication: Not applicable

Availability of data and materials: Not applicable

Competing interests: None declared.

Funding: This work was supported by a grant from National Science and Technology Major Project of the Ministry of Science and Technology of China (Grant number 2018ZX10306412-004).

Authors' contributions: All the authors have accepted responsibility for the entire content of this submitted manuscript and approved submission. LW and LC designed the study. LC, HJ and FG conducted the laboratory tests. HJ, FG and XJ collected and LC analyzed data and prepared the manuscript. All authors reviewed the manuscript and approved the final version of the manuscript and take responsibility for the integrity of the data and accuracy of data analysis.

Acknowledgements: We thank involved laboratory staff from blood centers or blood banks for participating in sample collection and testing.

\section{References}

1. Dore GJ, Hatzakis A, Negro F, Waked I. Estimating HCV disease burden-volume 4 (editorial). J Viral Hepat. 2017;24(Suppl 2):4-7.

2. Chen DS, Hamoudi W, Mustapha B, Layden J, Nersesov A, Reic T, et al. Strategies to manage hepatitis C virus infection disease burdenVolume 4. J Viral Hepat. 2017;24(Suppl 2):44-63.

3. Chan HLY, Chen CJ, Omede O, Al Qamish J, Al Naamani K, Bane A, et al. The present and future disease burden of hepatitis C virus infections with today's treatment paradigm: Volume 4. J Viral Hepat. 2017;24(Suppl 2):25-43.

4. Polaris Observatory HCVC. Global prevalence and genotype distribution of hepatitis C virus infection in 2015: a modelling study. Lancet Gastroenterol Hepatol. 2017;2(3):161-76.

5. Kamili S, Drobeniuc J, Araujo AC, Hayden TM. Laboratory diagnostics for hepatitis C virus infection. Clin Infect Dis. 2012;55(Suppl 1):43-8.

6. Kuroishi A, Yasui K, Matsukura H, Tani Y, Furuta RA. Comparison of neutralization profiles for anti-HCV reactive donor samples with or without detectable HCV RNA. Vox Sang. 2015;109(4):319-26.

7. Li L, Li KY, Yan K, Ou G, Li W, Wang J, et al. The History and Challenges of Blood Donor Screening in China. Transfus Med Rev. 2017;31(2):8993.

8. Mullis CE, Laeyendecker O, Reynolds SJ, Ocama P, Quinn J, Boaz I, et al. High frequency of false-positive hepatitis C virus enzyme-linked immunosorbent assay in Rakai, Uganda. Clin Infect Dis. 2013;57(12):1747-50.

9. Schroter M, Feucht HH, Schafer P, Zollner B, Polywka S, Laufs R. Definition of false-positive reactions in screening for hepatitis C virus antibodies. J Clin Microbiol. 1999;37(1):233-4. 
10. Majid AM, Gretch DR. Current and future hepatitis C virus diagnostic testing: problems and advancements. Microbes Infect. 2002;4(12):122736.

11. Seigneres B, Descamps F, Croise R, Barlet V, Bouvier-Alias M, Chevaliez S, et al. Multicenter clinical evaluation of the new 3rd generation assay for detection of antibodies against hepatitis C virus on the VIDAS((R)) system. J Clin Virol. 2016;78:20-6.

12. Hyun J, Ko DH, Kang HJ, Whang DH, Cha YJ, Kim HS. Evaluation of the VIDAS Anti-HCV Assay for Detection of Hepatitis C Virus Infection. Ann Lab Med. 2016;36(6):550-4.

13. Yoo SJ, Wang LL, Ning HC, Tao CM, Hirankarn N, Kuakarn S, et al. Evaluation of the Elecsys((R)) Anti-HCV II assay for routine hepatitis C virus screening of different Asian Pacific populations and detection of early infection. J Clin Virol. 2015;64:20-7.

14. Sommese L, Sabia C, Paolillo R, Parente D, Capuano M, lannone C, et al. Screening tests for hepatitis B virus, hepatitis C virus, and human immunodeficiency virus in blood donors: evaluation of two chemiluminescent immunoassay systems. Scand J Infect Dis. 2014;46(9):660-4.

15. Yang R, Guan W, Wang Q, Liu Y, Wei L. Performance evaluation and comparison of the newly developed Elecsys anti-HCV II assay with other widely used assays. Clin Chim Acta. 2013;426:95-101.

16. Esteban JI, van Helden J, Alborino F, Burgisser P, Cellerai C, Pantaleo G, et al. Multicenter evaluation of the Elecsys(R) anti-HCV II assay for the diagnosis of hepatitis C virus infection. J Med Virol. 2013;85(8):1362-8.

17. Berger A, Rabenau H, Allwinn R, Doerr HW. Evaluation of the new ARCHITECT anti-HCV screening test under routine laboratory conditions. J Clin Virol. 2008;43(2):158-61.

18. Watterson JM, Stallcup P, Escamilla D, Chernay P, Reyes A, Trevino SC. Evaluation of the Ortho-Clinical Diagnostics Vitros ECi Anti-HCV test: comparison with three other methods. J Clin Lab Anal. 2007;21(3):162-6.

19. Zachary P, Ullmann M, Djeddi S, Meyer N, Wendling MJ, Schvoerer E, et al. Evaluation of three commercially available hepatitis C virus antibody detection assays under the conditions of a clinical virology laboratory. J Clin Virol. 2005;34(3):207-10. discussion 16 - 8.

20. Oethinger M, Mayo DR, Falcone J, Barua PK, Griffith BP. Efficiency of the ortho VITROS assay for detection of hepatitis C virus-specific antibodies increased by elimination of supplemental testing of samples with very low sample-to-cutoff ratios. J Clin Microbiol. 2005;43(5):2477-80.

21. Kiely P, Styles C. Anti-HCV immunoblot indeterminate results in blood donors: non-specific reactivity or past exposure to HCV? Vox Sang. 2017;112(6):542-8.

\section{Figures}

\section{Figure 1}

Confirmatory results of plasma panel Note: +, POS: positive/reactive; -, NEG: negative/non-reactive; +/-, IND: indeterminate; R: Elecsys Anti-HCV II (Roche Diagnostics GmbH, Penzberg, Germany); A: ARCHITECT anti-HCV (Abbott, Illinois, US).

ras

\section{Figure 2}

Performance index of different assays Sensitivity (red), specificity (green), Youden's index (blue), and Kappa Coefficient (purple) of single assay and two combined assays were shown. The darker the color, the better performance the assays have. The number in the black box shows the best performance (sensitivity, specificity, Youden's index, or Kappa Coefficient) of all the combined assays. 


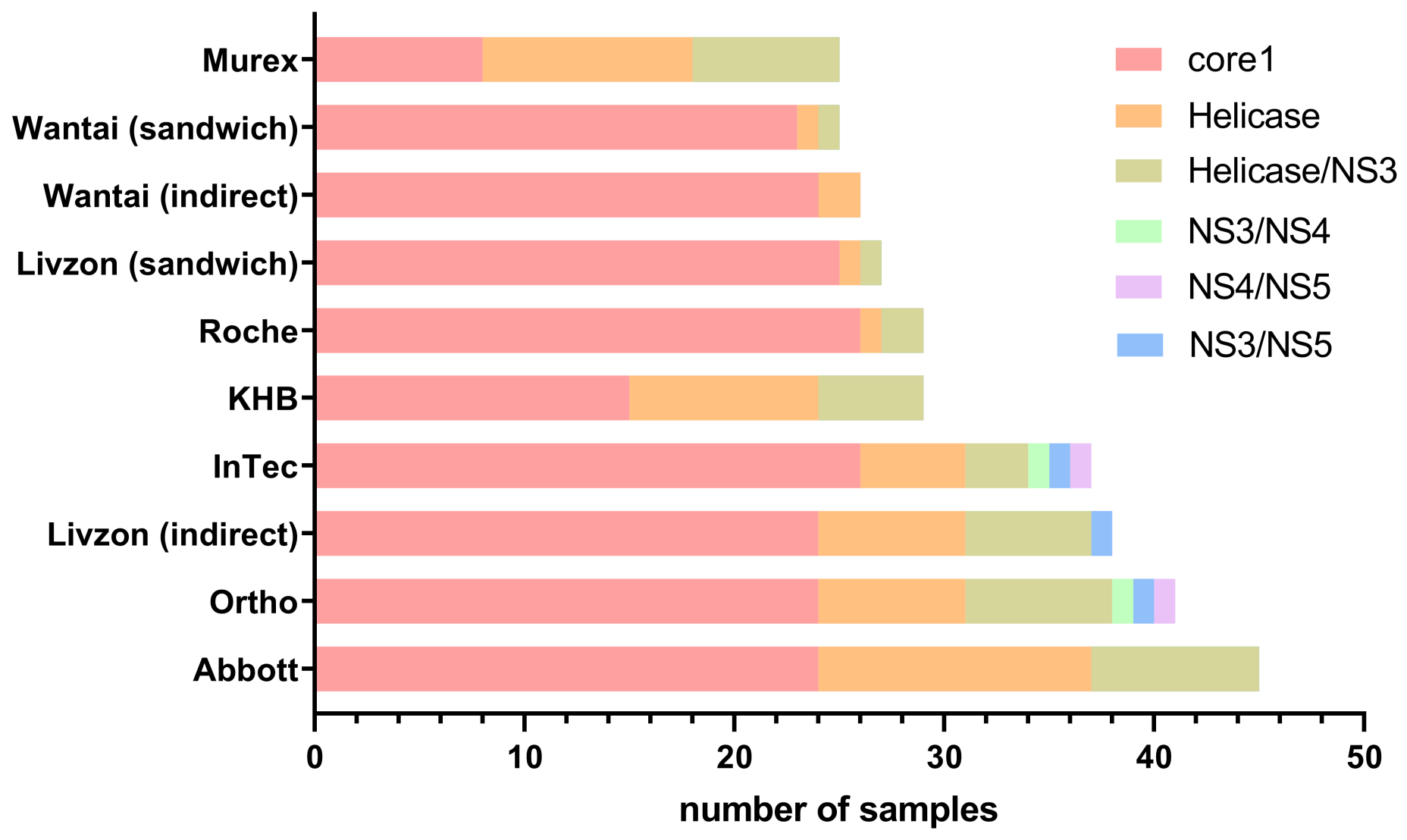

Figure 3

Antigen distribution on indeterminate samples of all the 85 indeterminate samples, different assays have different number of positive results (varies from 25 to 45) and distributions on different antigens (core1, helicase, helicase/NS3, NS3/NS4, NS3/NS5, and NS4/NS5). 


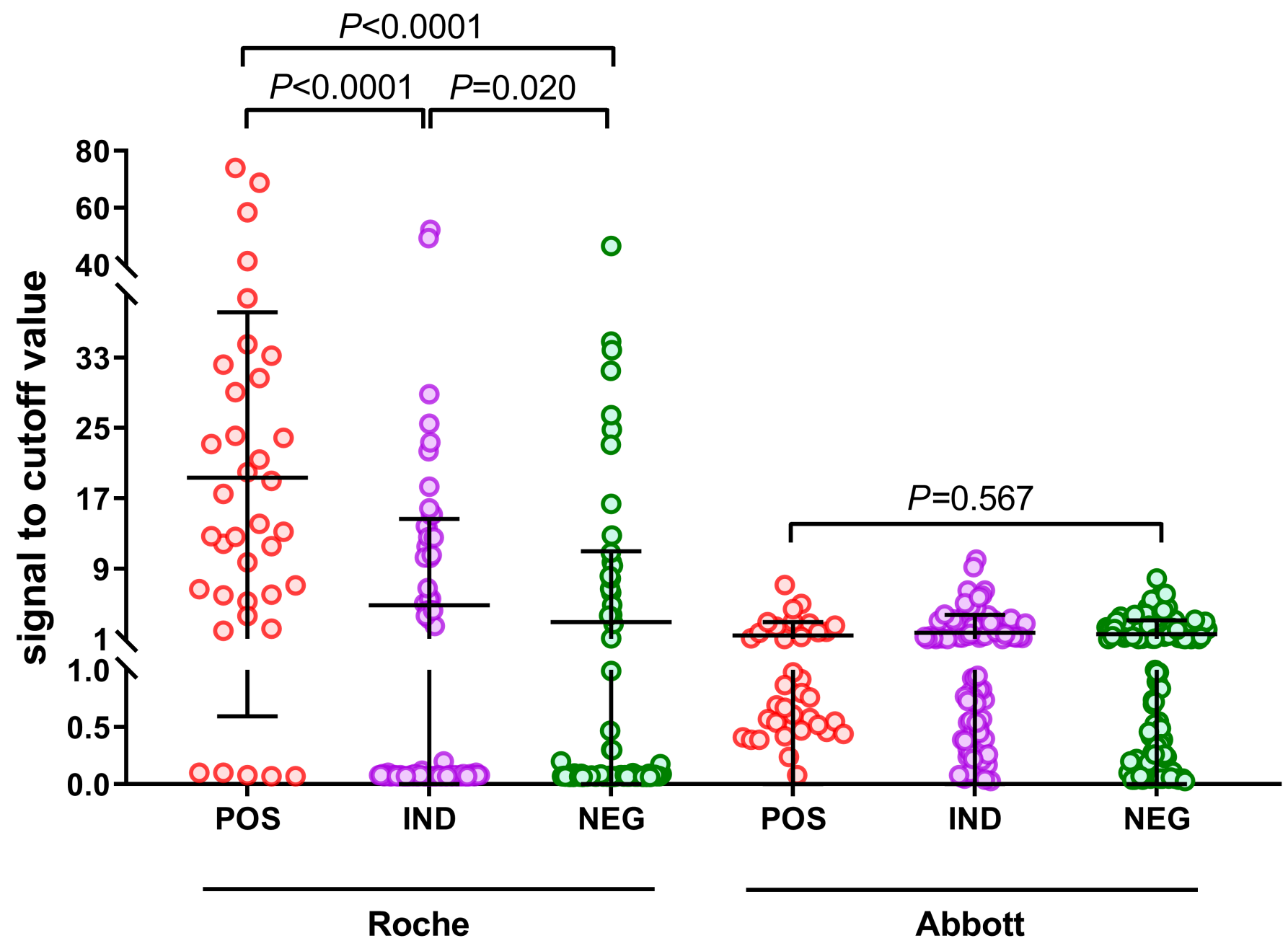

Figure 4

S/CO ratio distribution among confirmed positive, indeterminate and negative samples Note: +: positive/reactive; -: negative/non-reactive; +/-: indeterminate; Roche: Elecsys Anti-HCV II (Roche Diagnostics GmbH, Penzberg, Germany); Abbott: ARCHITECT anti-HCV (Abbott, Illinois, US). 